\title{
Cosmological General Theory of Relativity
}

\author{
Sangwha-Yi* \\ Department of Math, Taejon University 300-716, South Korea
}

*Corresponding Author: Sangwha-Yi, Department of Math, Taejon University 300-716, South Korea

\begin{abstract}
In expanded universe, we found gravity field equation and solution. We found Schwarzschild solution, Kerr-Newman solution in expanded universe. Hence, We found new general relativity theoryCosmological General Theory of Relativity(CGTR).

Keywords: Cosmological General Theory of Relativity; Newtonian Gravity; Schwarzschild solution; Kerr-
\end{abstract} Newman solution; Robertson-Walker solution

PACS Number: 04,04.90. $+e, 41.20$

\section{INTRODUCTION}

Our article's aim is that we make Cosmological General theory of Relativity (CGRT).

At first, Robertson-Walker metric is

$d \tau^{2}=d t^{2}-\frac{1}{c^{2}} \Omega^{2}(t)\left[\frac{d r^{2}}{1-k r^{2}}+r^{2} d \Omega^{2}\right]$

According to $\Lambda C D M$ model, our universe's $\mathrm{k}$ is zero. In this time, if $t_{0}$ is cosmological time,[2]

$k=0, t=t_{0}>\Delta t, \Delta t$ is period of matter's motion

Hence, the proper time is in cosmological time,

$$
\begin{aligned}
& d \tau^{2}=d t^{2}-\frac{1}{c^{2}} \Omega^{2}\left(t_{0}\right)\left[d r^{2}+r^{2} d \Omega^{2}\right] \\
& =d t^{2}-\frac{1}{c^{2}} \Omega^{2}\left(t_{0}\right)\left[d x^{2}+d y^{2}+d z^{2}\right] \\
& =d t^{2}\left(1-\frac{1}{c^{2}} \Omega^{2}\left(t_{0}\right) V^{2}\right), \quad V^{2}=\frac{d x^{2}+d y^{2}+d z^{2}}{d t^{2}}
\end{aligned}
$$

In this time,

$d \bar{t}=d t, d \bar{x}=\Omega\left(t_{0}\right) d x, d \bar{y}=\Omega\left(t_{0}\right) d y, d \bar{z}=\Omega\left(t_{0}\right) d z$

In Cosmological General theory of Relativity(CGTR)'s differential operators are

$\frac{1}{c} \frac{\partial}{\partial \bar{t}}=\frac{1}{c} \frac{\partial}{\partial t}, \frac{\partial}{\partial \bar{x}}=\frac{\partial}{\partial x} \frac{1}{\Omega\left(t_{0}\right)}, \frac{\partial}{\partial \bar{y}}=\frac{\partial}{\partial y} \frac{1}{\Omega\left(t_{0}\right)}, \frac{\partial}{\partial \bar{z}}=\frac{\partial}{\partial z} \frac{1}{\Omega\left(t_{0}\right)}$

Hence, 
$\frac{1}{c^{2}} \frac{\partial^{2}}{\partial \bar{t}^{2}}-\bar{\nabla}^{2}=\frac{1}{c^{2}} \frac{\partial^{2}}{\partial t^{2}}-\frac{1}{\Omega^{2}\left(t_{0}\right)}\left\{\left(\frac{\partial}{\partial x}\right)^{2}+\left(\frac{\partial}{\partial y}\right)^{2}+\left(\frac{\partial}{\partial z}\right)^{2}\right\}$

\section{NEWTONIAN GRAVITY IN EXPANDED UNIVERSE}

Newton's Gravity is built in static universe. Hence, for making our cosmological theory, we modified Newtonian Gravity in expanded universe.

At first, Newton's gravity acceleration is

$$
\begin{aligned}
& \overline{\vec{a}}=\vec{a} \Omega\left(t_{0}\right)=-\overline{\vec{\nabla}} \bar{\phi}=-\frac{1}{\Omega^{2}\left(t_{0}\right)} \vec{\nabla} \phi \\
& \bar{\phi}=\phi \frac{1}{\Omega\left(t_{0}\right)}=-\frac{G M}{I \Omega\left(t_{0}\right)}
\end{aligned}
$$

or

$$
\bar{\phi}=\phi \frac{1}{\Omega\left(t_{0}\right)}=\frac{1}{2} \frac{G M}{R^{3} \Omega^{3}\left(t_{0}\right)} r^{2} \Omega^{2}\left(t_{0}\right)=\frac{1}{2} \frac{G M}{R^{3}} r^{2} \frac{1}{\Omega\left(t_{0}\right)}
$$

In this time, if Newton's gravity potential is

$$
\bar{\phi}=\phi \frac{1}{\Omega\left(t_{0}\right)}=-\frac{G M}{r \Omega\left(t_{0}\right)}
$$

$\mathrm{Eq}(7)$ is

$$
\overrightarrow{\vec{a}}=\vec{a} \Omega\left(t_{0}\right)=-\frac{1}{\Omega^{2}\left(t_{0}\right)} \vec{\nabla} \phi=-\frac{G M}{r^{3}} \vec{r} \frac{1}{\Omega^{2}\left(t_{0}\right)}
$$

If Newton's gravity potential is

$$
\bar{\phi}=\phi \frac{1}{\Omega\left(t_{0}\right)}=\frac{1}{2} \frac{G M}{R^{3}} r^{2} \frac{1}{\Omega\left(t_{0}\right)}
$$

Poisson equation is in expanded universe,

$$
\bar{\nabla}^{2} \bar{\phi}=\frac{1}{\Omega^{3}\left(t_{0}\right)} \nabla^{2} \phi=4 \pi G \bar{\rho}, \bar{\rho}=\frac{\rho}{\Omega^{3}\left(t_{0}\right)}
$$

Newton force is in expanded universe,

$$
\overrightarrow{\vec{F}}=m_{0} \overline{\vec{a}}=m_{0} \vec{a} \Omega\left(t_{0}\right)=\vec{F} \Omega\left(t_{0}\right)
$$

\section{Cosmological General Theory of Relativity}

Einstein's geodesic equation is in expanded universe,

$$
\frac{d^{2} \bar{x}^{\mu}}{d \tau^{2}}+\bar{\Gamma}_{\alpha \beta}^{\mu} \frac{d \bar{x}^{\alpha}}{d \tau} \frac{d \bar{x}^{\beta}}{d \tau}=0
$$

Schwarzschild solution (vacuum solution) is in expanded universe,

$$
\begin{aligned}
& d s^{2}=-C^{2}\left(1-\frac{2 G M}{\bar{r} C^{2}}\right) d t^{2}+\frac{d \bar{r}^{2}}{1-\frac{2 G M}{\overline{r C}^{2}}}+\bar{r}^{2} d \theta^{2}+\bar{r}^{2} \sin ^{2} \theta d \varphi^{2} \\
& =-C^{2}\left(1-\frac{2 G M}{r \Omega\left(t_{0}\right) C^{2}}\right) d t^{2}+\Omega^{2}\left(t_{0}\right)\left[\frac{d r^{2}}{1-\frac{2 G M}{r \Omega\left(t_{0}\right) c^{2}}}+r^{2}\left(d \theta^{2}+\sin ^{2} \theta d \varphi^{2}\right)\right]
\end{aligned}
$$


Hence, Newtonian approximation is by $\mathrm{Eq}(11)$

$$
\bar{a}_{r}=\frac{d^{2} r}{d \tau^{2}} \Omega\left(t_{0}\right) \approx-\bar{\Gamma}_{00}^{1} c^{2}\left(\frac{d t}{d \tau}\right)^{2} \approx \frac{1}{2} c^{2} \frac{\partial \bar{g}_{00}}{\partial \bar{r}}=\frac{1}{2} \frac{c^{2}}{\Omega\left(t_{0}\right)} \frac{\partial g_{00}}{\partial r}=-\frac{G M}{r^{2}} \frac{1}{\Omega^{2}\left(t_{0}\right)}
$$

Hence, the gravity field equation of Einstein in expanded universe,

$\bar{R}_{\mu \nu}-\frac{1}{2} \bar{g}_{\mu \nu} \bar{R}=-\frac{8 \pi G}{C^{4}} \bar{T}_{\mu \nu}$

In this time,

$$
\bar{T}_{00}=\bar{\rho} C^{2}=\frac{\rho}{\Omega^{3}\left(t_{0}\right)} C^{2}=\frac{T_{00}}{\Omega^{3}\left(t_{0}\right)}
$$

Einstein's general solution- Kerr-Newman solution is in expanded universe,[1]

$$
\begin{aligned}
& d s^{2}=\bar{g}_{\mu \nu} d \bar{x}^{\mu} d \bar{x}^{v}=-c^{2}\left(1-\frac{2 C^{2} G M \bar{r}-k G Q^{2}}{c^{4} \bar{\Sigma}}\right) d t^{2}-2\left(2 c^{2} M G \bar{r}-k G Q^{2}\right) \frac{\bar{a} \sin ^{2} \theta}{c^{4} \bar{\Sigma}} c d t d \varphi \\
& +\frac{c^{4} \bar{\Sigma}}{\bar{r}^{2}-c^{2} 2 G M \bar{r}+\bar{a}^{2}+k G Q^{2}} d \bar{r}^{2}+\bar{\Sigma} d \theta^{2} \\
& +\sin ^{2} \theta\left[\bar{r}^{2}+\bar{a}^{2}+\left(2 c^{2} G M \bar{r}-k G Q^{2}\right) \frac{\bar{a}^{2} \sin ^{2} \theta}{c^{4} \bar{\Sigma}}\right] d \varphi^{2} \\
& \bar{\Sigma}=\bar{r}^{2}+\bar{a}^{2} \cos ^{2} \theta=\left(r^{2}+a^{2} \Omega^{2}\left(t_{0}\right) \cos ^{2} \theta\right) \Omega^{2}\left(t_{0}\right) \\
& =\Sigma \Omega^{2}\left(t_{0}\right), \quad \Sigma '=r^{2}+a^{2} \Omega^{2}\left(t_{0}\right) \cos ^{2} \theta
\end{aligned}
$$

Hence, Kerr-Newman solution is expanded universe,

$$
\begin{aligned}
& d s^{2}=\bar{g}_{\mu \nu} d \bar{x}^{\mu} d \bar{x}^{v}=-c^{2}\left(1-\frac{2 c^{2} G M I \Omega\left(t_{0}\right)-k G Q^{2}}{c^{4} \Sigma \Omega^{2}\left(t_{0}\right)}\right) d t^{2} \\
& -2\left(2 c^{2} M G I \Omega\left(t_{0}\right)-k G Q^{2}\right) \frac{a \Omega^{2}\left(t_{0}\right) \sin ^{2} \theta}{c^{4} \Sigma \Omega^{2}\left(t_{0}\right)} c d t d \varphi \\
& +\Omega^{2}\left(t_{0}\right)\left\{\frac{c^{4} \Sigma \Omega^{2}\left(t_{0}\right)}{r^{2} \Omega^{2}\left(t_{0}\right)-c^{2} 2 G M I \Omega\left(t_{0}\right)+a^{2} \Omega^{4}\left(t_{0}\right)+k G Q^{2}} d r^{2}+\Sigma ' d \theta^{2}\right. \\
& \left.\left.+\sin ^{2} \theta\left[r^{2}+a^{2} \Omega^{2}\left(t_{0}\right)+R c^{2} G M I \Omega\left(t_{0}\right)-k G Q^{2}\right) \frac{a^{2} \Omega^{4}\left(t_{0}\right) \sin ^{2} \theta}{\Omega^{4}\left(t_{0}\right) c^{4} \Sigma{ }^{\prime}}\right] d \varphi^{2}\right\} \\
& \bar{\Sigma}=\bar{r}^{2}+\bar{a}^{2} \cos ^{2} \theta=\left(r^{2}+a^{2} \Omega^{2}\left(t_{0}\right) \cos ^{2} \theta\right) \Omega^{2}\left(t_{0}\right) \\
& =\Sigma \Omega^{2}\left(t_{0}\right), \quad \Sigma '=r^{2}+a^{2} \Omega^{2}\left(t_{0}\right) \cos s^{2} \theta
\end{aligned}
$$

Robertson-Walker solution is Minkowski space-time by Einstein gravity field equation in CGTR- 
$\mathrm{Eq}(18)$ in expanded universe.

$$
d s^{2}=-c^{2} d t^{2}+\left[d \bar{r}^{2}+\bar{r}^{2} d \Omega^{2}\right]=-c^{2} d t^{2}+\Omega^{2}\left(t_{0}\right)\left[d r^{2}+r^{2} d \Omega^{2}\right]
$$

$\mathrm{Eq}(22)$ is equal to $\mathrm{Eq}(3) . \mathrm{Eq}(1)$ is derived by normal Einstein gravity field equation.

According to this theory, the distance traveled by light is changed by cosmological time.

If $x_{t_{2}-t_{1}}$ is the distance traveled by light during the time $t_{2}-t_{1}$ in present cosmological time $t_{0}$,

$$
\Omega\left(t_{0}\right) x_{t_{2}-t_{1}}=x_{t_{2}-t_{1}}=C\left(t_{2}-t_{1}\right), \quad \Omega\left(t_{0}\right)=1
$$

If $x_{t_{0}-\Delta t+t_{2}-t_{1}}$ is the distance traveled by light in the past universe,

$$
\Omega\left(t_{0}-\Delta t\right) x_{t_{0}-\Delta t+t_{2}-t_{1}}=C t_{t_{0}-\Delta t+t_{2}-t_{1}}=C\left(t_{2}-t_{1}\right)
$$

If $x_{t_{0}+\Delta t+t_{2}-t_{1}}$ is the distance traveled by light in the future universe,

$$
\Omega\left(t_{0}+\Delta t\right) x_{t_{0}+\Delta t+t_{2}-t_{1}}=C t_{t_{0}+\Delta t+t_{2}-t_{1}}=C\left(t_{2}-t_{1}\right)
$$

Universe is expanded. Hence,

$$
\Omega\left(t_{0}-\Delta t\right)<\Omega\left(t_{0}\right)=1<\Omega\left(t_{0}+\Delta t\right)
$$

Therefore, the distance traveled by light in the past universe is longer than the distance traveled by light in the future universe.

$$
x_{t_{0}-\Delta t+t_{2}-t_{1}}>x_{t_{2}-t_{1}}>x_{t_{0}+\Delta t+t_{2}-t_{1}}
$$

\section{CONCLUSiON}

We find Cosmological General theory of Relativity. We obtain solution of Einstein gravity field equation in expanded universe..

\section{REFERENCES}

[1]S.Yi, "PMBH Theory of Representation of Gravity Field Equation and Solution, Hawking Radiation in Data General Relativity Theory", International Journal of Advanced Research in Physical Science,5,9,(2018),pp36-45 [2]S.Yi, "Cosmological Special Theory of Relativity", International Journal of Advanced Research in Physical Science, 7,11,(2020),pp4-9

[3]S.Yi, "Spherical Solution of Classical Quantum Gravity", International Journal of Advanced Research in Physical Science,6,8,(2019),pp3-6

[4]A. Einstein, “ Zur Elektrodynamik bewegter K"orper”, Annalen der Physik. 17:891(1905)

[5]Friedman-Lemaitre-Robertson-Walker metric-Wikipedia

[6]A.Miller, Albert Einstein's Special Theory of Relativity(Addison-Wesley Publishing Co., Inc., 1981)

[7]Lambda-CDM model -Wikipedia

[8]S. Weinberg, Gravitation and Cosmology(John wiley \& Sons,Inc,1972)

[9]C. Misner, K, Thorne and J. Wheeler, Gravitation (W.H.Freedman \& Co.,1973) 
[10]S. Hawking and G. Ellis, The Large Scale Structure of Space-Time(Cam-bridge University Press,1973)

[11]R. Adler,M.Bazin and M.Schiffer,Introduction to General Relativity(McGraw-Hill,Inc.,1965)

[12]P. Roll, R. Krotkov and R. Dicke, Ann. Phys.(U.S.A) 26,442(1964)

[13]V. Braginsky and V. Panov, Zh. Eksp. \& Teor. Fiz.61, 873(1971)(English translation, Sov. Phys.-JETP 34,464(1971)

[14]L.Einsenhart, Riemannian Geometry(Princeton University Press, 1926)

[15]J. Schouten, Ricci-Calculus(Springer-Verlag, Berlin, 1954)

[16]A. Einstein,'The Foundation of General Relativity", Ann. Phys. (Germany)49(1916)

[17]G.Birkoff, Relativity and Modern Physics (Harvard University Press,1923),p253

[18]A.Raychaudhuri, Theorical Cosmology(Oxpord University Press,1979)

[19]E. Kasner, Am. J. Math. 43, 217(1921)

[20]D.N.Page. ”Hawking Radiation and Black hole Thermodynamics.”.arXiv:hep-th/040924

[21]M.Rabinowitz."Gravitaational Tunneling Radiation”. Physics Essays. 12(2):346-357.arxiv:astro$\mathrm{ph} / 0212249(2000)$

[22]S,Giddings and S.Thomas "High energy colliders as black hole factories: The end of short distant physics". Physical Review D. 65(5)(2002)

[23]S.Dimoploulus and G.Landsberg "Black holes at the Large Hardron Collider". Physical Review Letter. 87(16):161602.arxiv:hep-th/0106295(2001)

[24]F.Belgiorno ,S.Cacciatori, M.Clerici, V.Gorini, G. Ortenzi, L.Rizzi ,E.Rubbino, V.Sala D. Faccio.”Hawking Radiation from ultrashort laser pulse filaments”.Phys. Rev. Lett. 105(20):203901(2010):arxiv:1009.4634

[25]K. Kumar,B. Kiranagi,C.Begewadi,’Hawking Radiation- An Augmentation Attrition Model”.Adv.Nat.Sci.5.(2):14-33(2012)

[26]A.Helfer"Do black-holes radiate?".Reports on Progress in Physics. 66(6):943-1008(2003):arxiv:grqc/0304042.(2003)

[27]R.Brout,S.Massar,R.Parentani,P.Spindel"Hawking radiation without tras-Plankian frequencies".Phiscal Review D.52(8):4559-4568(1995):arxiv:hep-th/9506121

[28]D.Page,"Particle emission rates from a black-hole:Massless particles from an unchrge,nonrotating hole”.Physical Review D.13(2):198-206(1976)

[29]T.Jacobson,'Black hole-evaporation and ultrashorts distances” Physical Review D.44(6):1731-1739(1991)

[30]J.Kapusta,"The Last Eight Minutes of a Primordial Black-hole"(1999):Arxiv:astro-ph/9911309

[31]A.Ashtekar,J.Baez,A.Corichi,K.Krasnov,"'Quantum Geometry and Hole Entropy”Phys.Rev.Lett.80(5):904-907(1998):arxiv:gr-qc/9710007

Citation: Sangwha-Yi (2021). Cosmological General Theory of Relativity. International Journal of Advanced Research in Physical Science (IJARPS) 8(2), pp.22-26, 2021.

Copyright: (C) 2021 Authors, This is an open-access article distributed under the terms of the Creative Commons Attribution License, which permits unrestricted use, distribution, and reproduction in any medium, provided the original author and source are credited. 